\title{
Quantification of Bio-oil Functional Groups and Evidences of the Presence of Pyrolytic Humins
}

\section{Supporting Information}
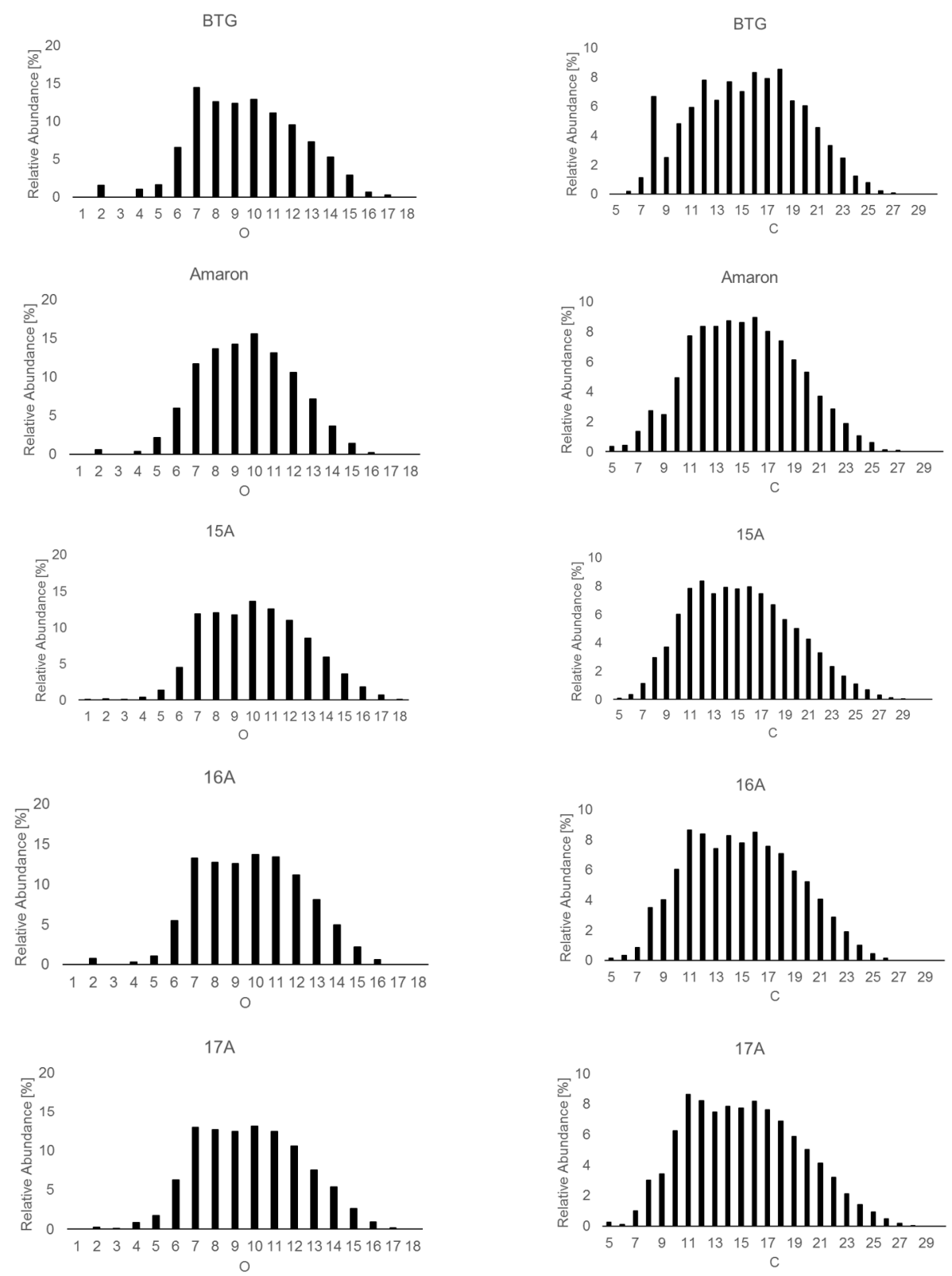

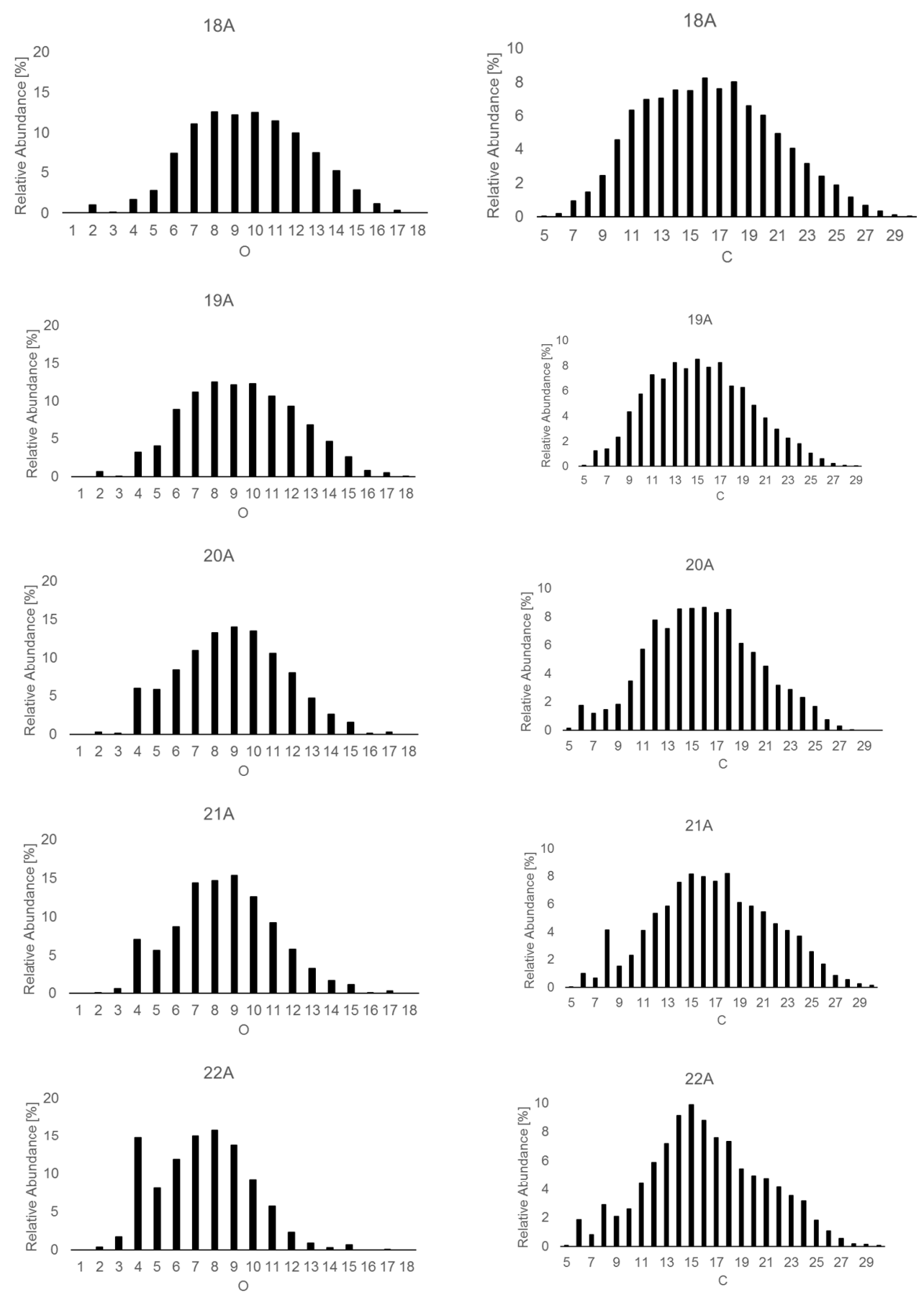

Figure S1. Heteroatom class distribution for the data obtained by FT-ICR-MS 

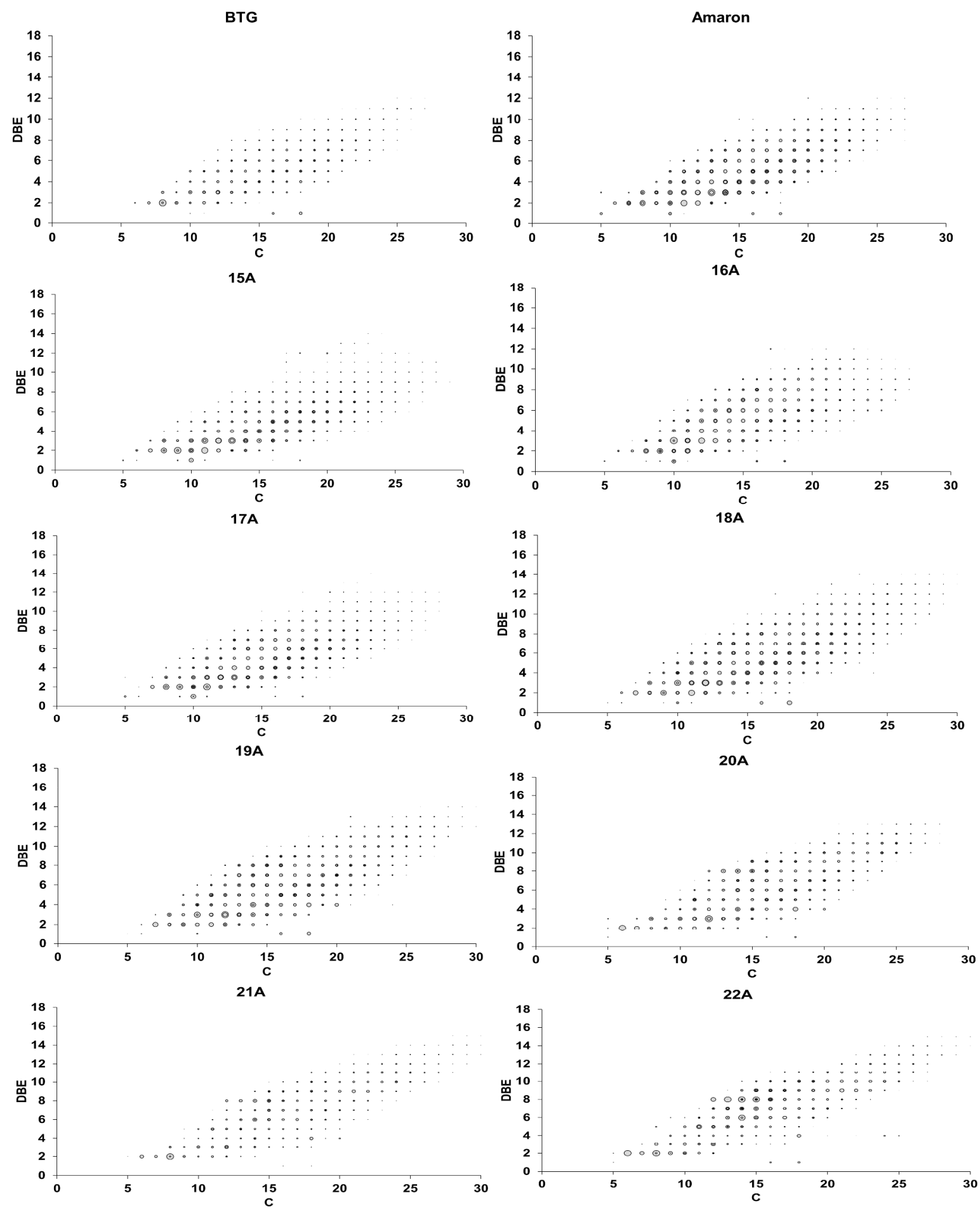
Figure S2. DBE vs. C number from the data obtained by FT-ICR-MS 

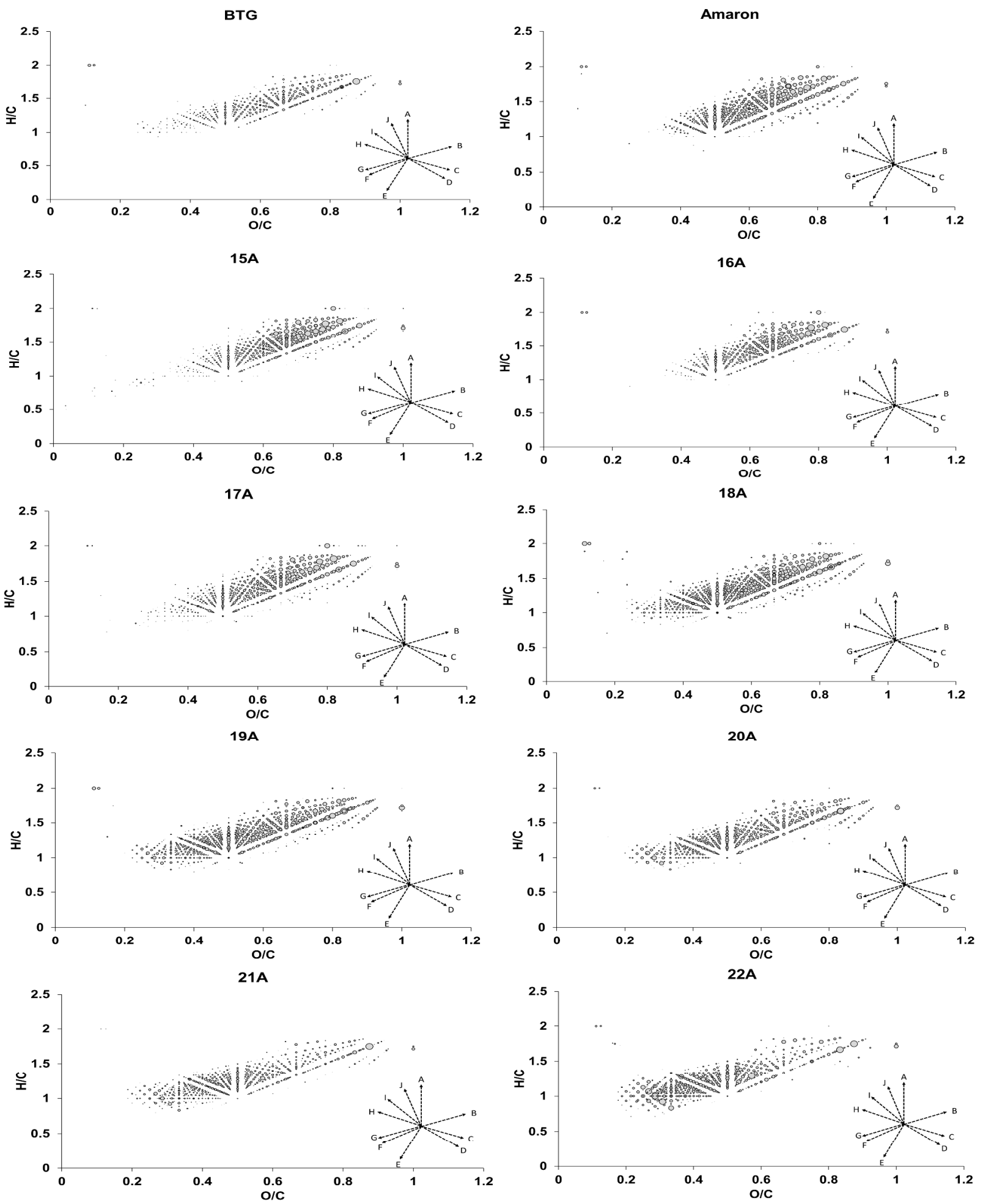

Figure S3. Van Krevelen plot of the non-volatile fraction of the bio-oils. Distinctive line patterns can be associated with: loss of levoglucosan/hydration (A), loss of furan (B), loss of acetol (D), demethoxylation (E), dehydration (F), loss of glycolaldehyde/acetic acid (G), deraboxylation $(\mathrm{H})$, decarbonylation $(\mathrm{J})$. 


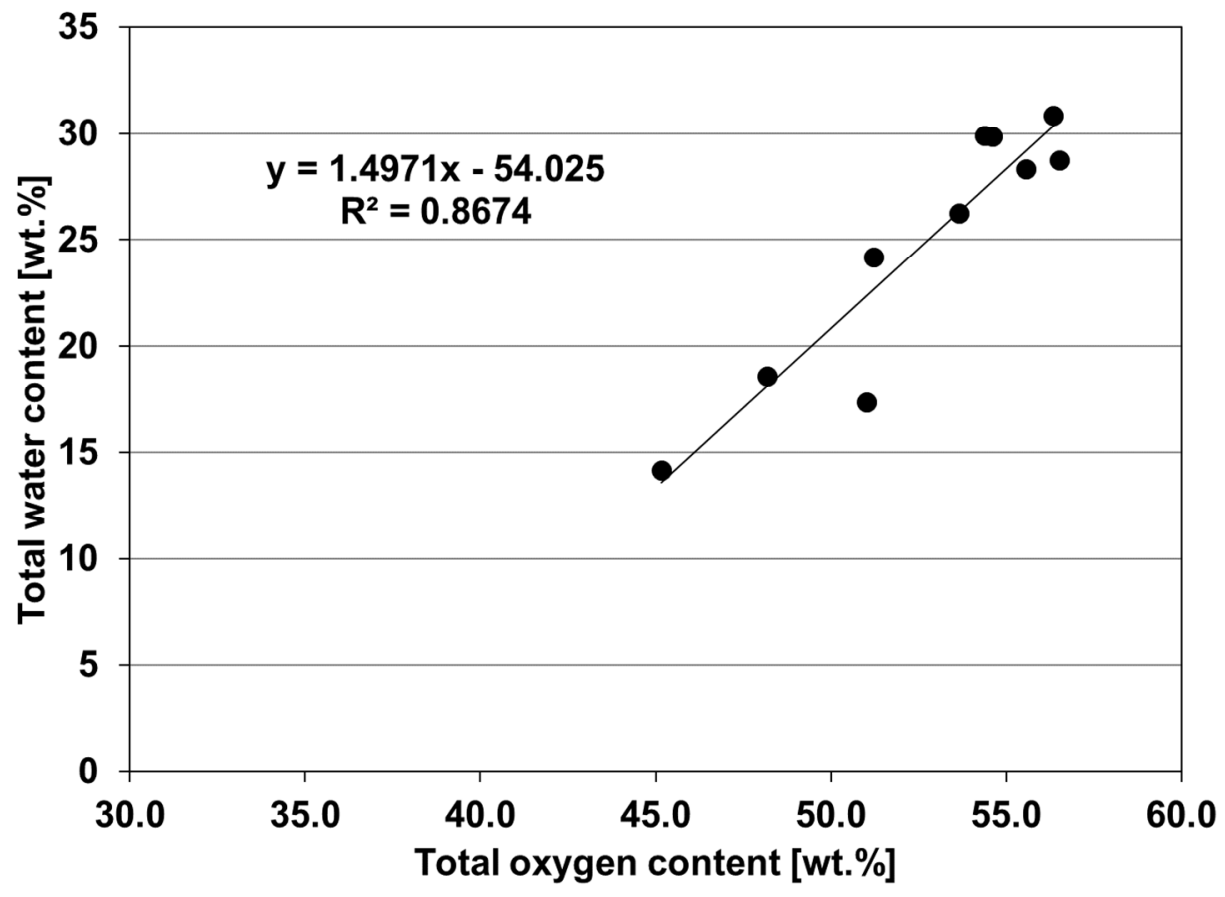

Figure S4. Correlation between bio-oil total oxygen and water content 


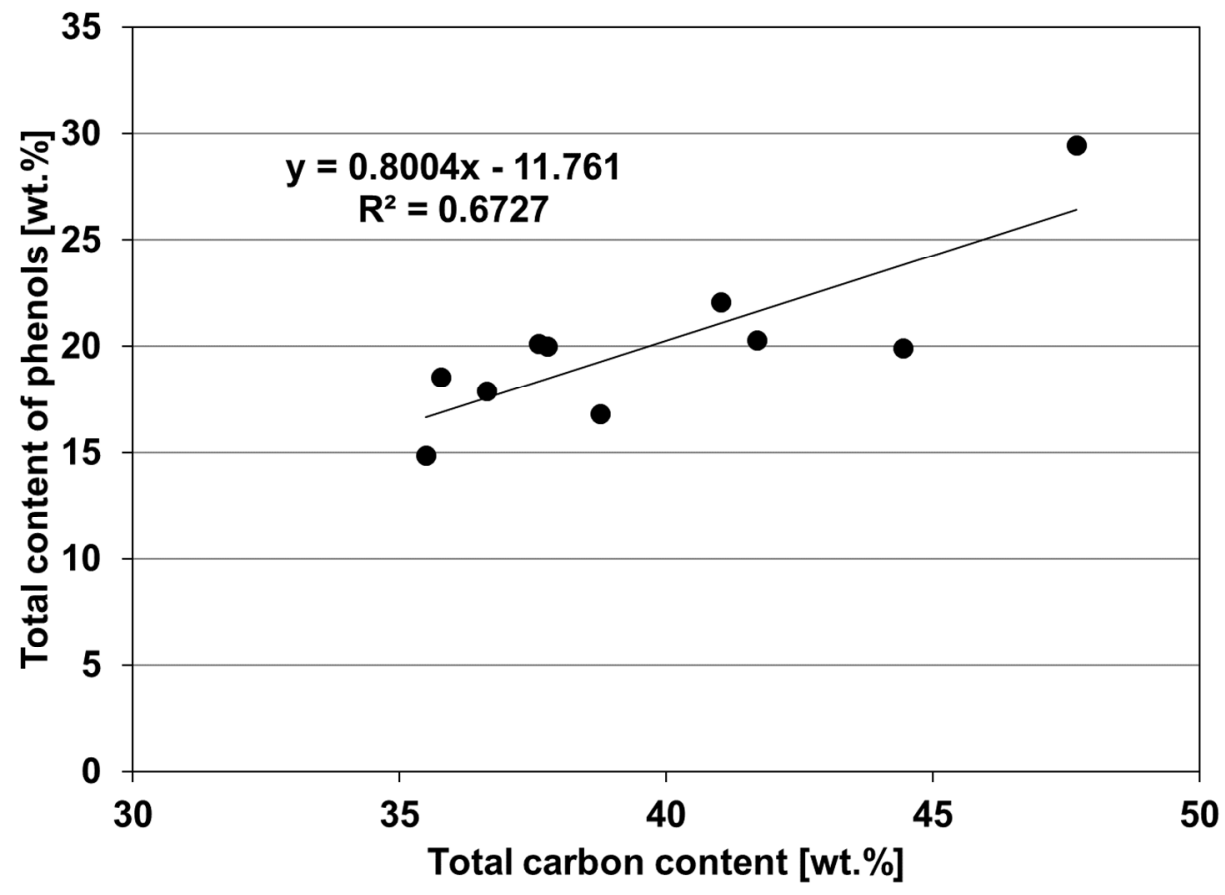

Figure S5. Correlation between the content of total phenols and total carbon content.

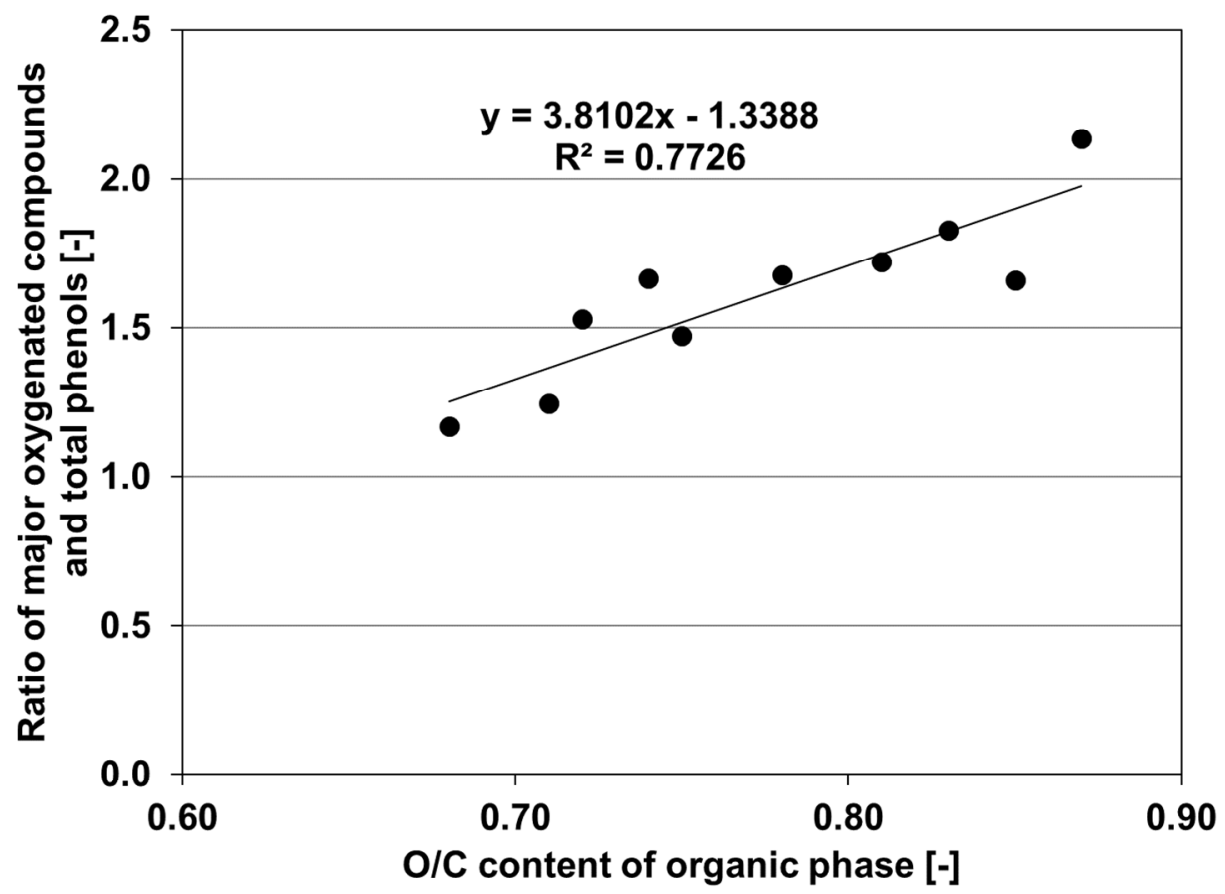

Figure S6. Correlation between the ratio of major oxygenated compounds (total sugar + acetic acid + hydroxydataldehyde + acetol) to total phenol content and the $\mathrm{O} / \mathrm{C}$ ratio of the organic phase 


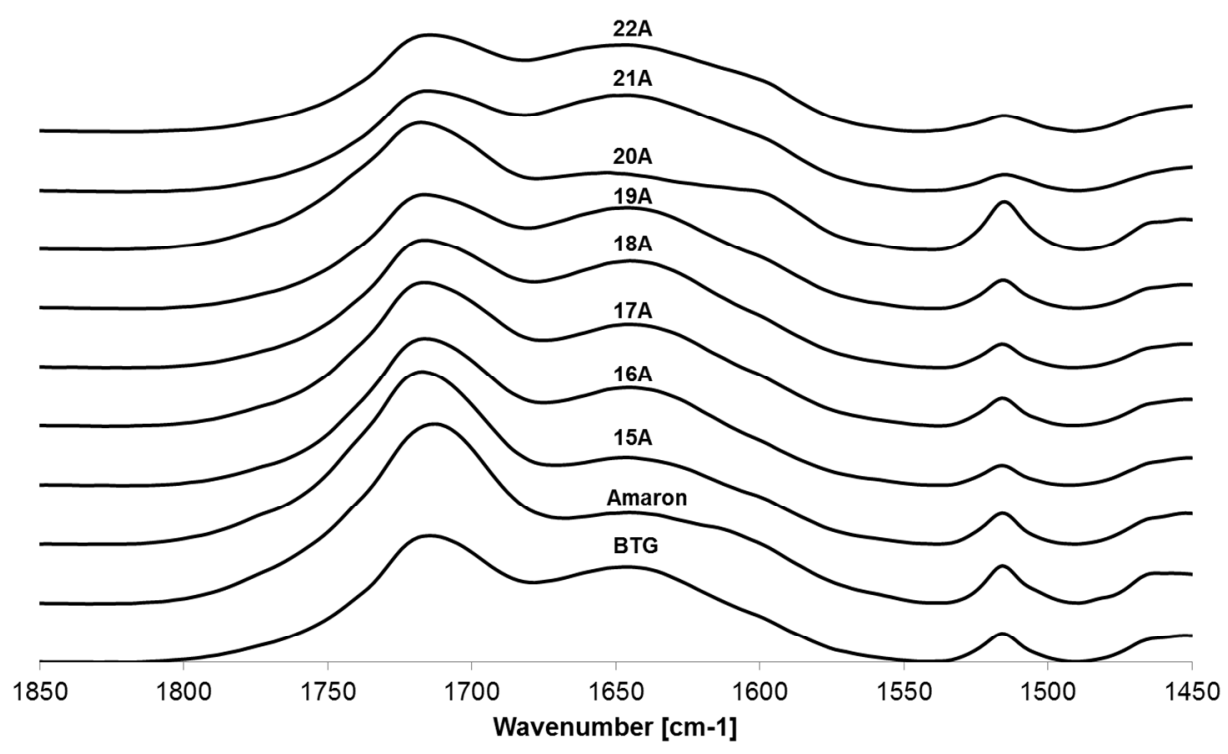

Figure S7. FTIR spectra of bio-oils in the region characteristic for carbonyl group vibrations and for aromatic breathing frequency 
Table S1. Number-, weight-, z-average molecular weight, polydispersity index and number of identified compounds of the heavy fraction of bio-oils

\begin{tabular}{|c|c|c|c|c|c|}
\hline Sample & $\mathrm{Mn}$ & $\mathrm{Mw}$ & $\mathrm{Mz}$ & Polydispersity & $\begin{array}{c}\text { Identified } \\
\text { compounds }\end{array}$ \\
\hline BTG & 359.3 & 383.2 & 405.0 & 1.07 & 439 \\
\hline Amaron & 356.9 & 377.5 & 396.4 & 1.06 & 478 \\
\hline 15A & 369.0 & 394.1 & 417.6 & 1.07 & 616 \\
\hline 16A & 358.8 & 381.4 & 402.3 & 1.06 & 413 \\
\hline 17A & 361.1 & 384.6 & 406.6 & 1.07 & 613 \\
\hline 18A & 372.1 & 396.1 & 418.3 & 1.06 & 740 \\
\hline 19A & 366.5 & 389.5 & 410.8 & 1.06 & 697 \\
\hline 20A & 353.4 & 375.3 & 395.0 & 1.06 & 555 \\
\hline 21A & 358.3 & 381.2 & 401.9 & 1.06 & 631 \\
\hline 22A & 331.0 & 353.3 & 374.2 & 1.07 & 564 \\
\hline
\end{tabular}

Table S2. Elemental (C, H, N and O) composition of bio-oil (wt.\%)

\begin{tabular}{|c|c|c|c|c|}
\hline Sample & $\begin{array}{c}\mathrm{C} \\
{[\mathrm{wt.} \%]}\end{array}$ & $\begin{array}{c}\mathrm{H} \\
{[\mathrm{wt.} \%]}\end{array}$ & $\begin{array}{c}\mathrm{N} \\
{[\mathrm{wt.} \%]}\end{array}$ & $\begin{array}{c}\text { O* } \\
{[\mathrm{wt} . \%]}\end{array}$ \\
\hline BTG & 38.8 & 7.5 & 0.1 & 53.6 \\
\hline Amaron & 44.4 & 7.2 & 0.2 & 48.2 \\
\hline 15A & 41.7 & 7.3 & 0.0 & 51.0 \\
\hline $16 \mathrm{~A}$ & 35.5 & 8.0 & 0.0 & 56.5 \\
\hline $17 \mathrm{~A}$ & 36.6 & 7.8 & 0.0 & 55.6 \\
\hline $18 \mathrm{~A}$ & 35.8 & 7.9 & 0.0 & 56.4 \\
\hline 19A & 37.8 & 7.8 & 0.0 & 54.4 \\
\hline 20A & 47.7 & 7.2 & 0.0 & 45.2 \\
\hline $21 \mathrm{~A}$ & 37.6 & 7.8 & 0.0 & 54.6 \\
\hline 22A & 41.0 & 7.8 & 0.0 & 51.2 \\
\hline
\end{tabular}

*By difference 
Table S3. Characterization of pyrolysis oils functional groups by select spectral regions by ${ }^{1} \mathrm{H}-$ NMR with water suppression CPMG [\%] (Region Area [\%])

\begin{tabular}{|c|c|c|c|c|c|c|}
\hline $\begin{array}{c}\text { Spectral } \\
\text { region }\end{array}$ & $\begin{array}{c}0.5-1.5 \\
\mathrm{ppm}\end{array}$ & $\begin{array}{c}1.5-3.0 \\
\mathrm{ppm}\end{array}$ & $\begin{array}{c}3.0-4.2 \\
\mathrm{ppm}\end{array}$ & $\begin{array}{c}4.2-6.0 \\
\mathrm{ppm}\end{array}$ & $\begin{array}{c}6.0-9.2 \\
\mathrm{ppm}\end{array}$ & $\begin{array}{c}9.2-10.1 \\
\mathrm{ppm}\end{array}$ \\
\hline $\begin{array}{c}\text { Groups } \\
\text { assigned }\end{array}$ & Alkanes & $\begin{array}{c}\text { Aliphatics } \alpha \text {-to } \\
\text { heteroatom or } \\
\text { unsaturation }\end{array}$ & $\begin{array}{c}\text { Alcohols, } \\
\text { methylene } \\
\text {-benzene }\end{array}$ & $\begin{array}{c}\text { Methoxy, } \\
\text { carbohydrates }\end{array}$ & $\begin{array}{c}\text { (Hetero-) } \\
\text { aromatics }\end{array}$ & Aldehydes \\
\hline BTG & 10.4 & 27.7 & 36.9 & 13.3 & 11.2 & 0.6 \\
\hline Amaron & 16.0 & 36.2 & 30.8 & 6.6 & 10.0 & 0.4 \\
\hline $15 \mathrm{~A}$ & 9.1 & 29.9 & 36.8 & 13.6 & 10.0 & 0.7 \\
\hline $16 \mathrm{~A}$ & 11.4 & 29.8 & 36.5 & 12.8 & 8.8 & 0.6 \\
\hline $17 \mathrm{~A}$ & 9.2 & 30.9 & 35.3 & 12.6 & 11.4 & 0.7 \\
\hline $18 \mathrm{~A}$ & 9.3 & 30.5 & 35.7 & 12.7 & 11.2 & 0.6 \\
\hline $19 \mathrm{~A}$ & 10.1 & 27.6 & 35.1 & 12.3 & 14.2 & 0.8 \\
\hline $20 \mathrm{~A}$ & 9.5 & 27.1 & 35.0 & 12.7 & 15.0 & 0.7 \\
\hline $21 \mathrm{~A}$ & 9.7 & 25.0 & 33.1 & 15.5 & 15.8 & 0.9 \\
\hline $2 \mathrm{~A}$ & 11.9 & 24.6 & 31.2 & 14.8 & 16.7 & 0.8 \\
\hline
\end{tabular}

Table S4. Absorbance at $3400 \mathrm{~cm}^{-1}$ and $3200 \mathrm{~cm}^{-1}$

\begin{tabular}{|l|c|c|c|c|c|c|c|c|c|c|}
\hline & $\begin{array}{c}\text { BTG } \\
\text { Oil }\end{array}$ & $\begin{array}{c}\text { Amaron } \\
\text { Oil }\end{array}$ & $15 \mathrm{~A}$ & $16 \mathrm{~A}$ & $17 \mathrm{~A}$ & $18 \mathrm{~A}$ & $19 \mathrm{~A}$ & $20 \mathrm{~A}$ & $21 \mathrm{~A}$ & $22 \mathrm{~A}$ \\
\hline $3400 \mathrm{~cm}-1$ & 0.09 & 0.06 & 0.07 & 0.08 & 0.08 & 0.09 & 0.09 & 0.06 & 0.09 & 0.08 \\
\hline $3200 \mathrm{~cm}-1$ & 0.05 & 0.04 & 0.04 & 0.05 & 0.05 & 0.06 & 0.06 & 0.04 & 0.06 & 0.05 \\
\hline
\end{tabular}

Table S5. Fluorescence emission intensity at the three distinct peaks in the UV fluorescence spectra of the bio-oils diluted at $20 \mathrm{mg} / \mathrm{L}$ in methanol

\begin{tabular}{|c|c|c|c|c|c|}
\hline \multirow{2}{*}{ Sample } & $288 \mathrm{~nm}$ & $328 \mathrm{~nm}$ & $354 \mathrm{~nm}$ & \multirow{2}{*}{$\begin{array}{c}\text { Sum of } \\
\text { intensities }\end{array}$} & $\begin{array}{c}\text { Area in } \\
\text { arbitrary } \\
\text { units }\end{array}$ \\
\cline { 2 - 4 } & \multicolumn{3}{|c|}{ Signal intensity } & 14.1 & 260 \\
\hline BTG & 7.9 & 4.8 & 1.5 & 15.0 & 272 \\
\hline Amaron & 7.4 & 6.8 & 0.8 & 15.1 & 269 \\
\hline $15 \mathrm{~A}$ & 7.4 & 6.8 & 0.8 & 8.7 & 152 \\
\hline $16 \mathrm{~A}$ & 4.8 & 2.8 & 1.0 & 13.9 & 242 \\
\hline $17 \mathrm{~A}$ & 6.5 & 5.3 & 2.1 & 15.7 & 285 \\
\hline $18 \mathrm{~A}$ & 7.0 & 6.6 & 2.2 & 19.9 & 382 \\
\hline $19 \mathrm{~A}$ & 8.2 & 8.6 & 3.1 & 27.7 & 558 \\
\hline $20 \mathrm{~A}$ & 10.3 & 12.5 & 4.9 & 14.0 & 308 \\
\hline $21 \mathrm{~A}$ & 7.2 & 5.2 & 1.6 & 17.4 & 398 \\
\hline $22 \mathrm{~A}$ & 9.3 & 5.9 & 2.3 & & \\
\hline
\end{tabular}

